\title{
Plaque microstructures during metformin therapy in type 2 diabetic subjects with coronary artery disease: optical coherence tomography analysis
}

\author{
Yu Kataoka ${ }^{1}$, Stephen J. Nicholls ${ }^{2}$, Jordan Andrews ${ }^{3}$, Kiyoko Uno ${ }^{4}$, Samir R. Kapadia ${ }^{5}$ E. Murat Tuzcu ${ }^{5}$, \\ Steven E. Nissen ${ }^{5}$, Rishi Puri, \\ ${ }^{1}$ Department of Cardiovascular Medicine, National Cerebral \& Cardiovascular Center, Suita, Japan; ${ }^{2}$ Monash Cardiovascular Research Centre, \\ Victorian Heart Institute, Monash University, Melbourne, Australia; ${ }^{3}$ South Australian Health \& Medical Research Institute, University of Adelaide, \\ Adelaide, Australia; ${ }^{4}$ Teikyo Academic Research Center, Teikyo University, Tokyo, Japan; ${ }^{5}$ Department of Cardiovascular Medicine, Cleveland Clinic, \\ Cleveland, Ohio, USA; ${ }^{6}$ Cleveland Clinic Coordinating Center for Clinical Research, Cleveland, Ohio, USA \\ Contributions: (I) Conception and design: Y Kataoka, SJ Nicholls; (II) Administrative support: SJ Nicholls; (III) Provision of study materials or \\ patients: Y Kataoka, SJ Nicholls; (IV) Collection and assembly of data: Y Kataoka, R Puri; (V) Data analysis and interpretation: Y Kataoka, SJ \\ Nicholls; (VI) Manuscript writing: All authors; (VII) Final approval of manuscript: All authors. \\ Correspondence to: Stephen J. Nicholls. Monash Cardiovascular Research Centre. 246 Clayton Road, Clayton, VIC 3168 , Australia. \\ Email: stephen.nicholls@monash.edu.
}

Background: While metformin is recommended as a first-line cardioprotective therapy for type 2 diabetic patients, whether it exerts direct effects on atherosclerotic plaque remains uncertain. The current study characterized coronary plaque microstructures in type 2 diabetic patients who received metformin.

Methods: We retrospectively analyzed 409 non-culprit lipid plaques in 313 type 2 diabetic patients with coronary artery disease (CAD) by using frequency-domain optical coherence tomography (FD-OCT) imaging. FD-OCT derived plaque microstructures were compared in patients stratified according to metformin use.

Results: A proportion of $38.6 \%$ of study subjects received metformin. Patients receiving metformin more likely exhibited a history of hypertension $(79.3 \%$ vs. $67.1 \%, \mathrm{P}=0.03)$ and metabolic syndrome $(52.8 \%$ vs. $36.4 \%, \mathrm{P}=0.01)$. On FD-OCT imaging, the prevalence of lipid plaque was lower in the metformin group (66.2\% vs. $77.9 \%, \mathrm{P}=0.03)$. Furthermore, the metformin group demonstrated plaques with a smaller lipid arc (median: $168.7^{\circ}$ vs. $208.5^{\circ}, \mathrm{P}=0.008$ ), shorter longitudinal length (media: 5.1 vs. $9.1 \mathrm{~mm}, \mathrm{P}=0.04$ ), and a lower frequency of cholesterol crystal $(3.9 \%$ vs. $18.2 \%, \mathrm{P}=0.01)$ and spotty calcification $(3.9 \%$ vs. $34.8 \%, \mathrm{P}=0.008)$. These differences remained significant after adjusting for clinical characteristics and glycemic control. However, in patients who received insulin, the favourable effect of metformin on lipid arc was not observed (insulin user: $\mathrm{P}=0.87$; insulin non-user: $\mathrm{P}=0.009$; $\mathrm{P}$ value for interaction between two groups, $\mathrm{P}=0.02$ ).

Conclusions: Metformin use was associated with a lower prevalence of vulnerable plaque features in type 2 diabetic patients with $\mathrm{CAD}$, especially insulin non-user. These findings suggest the potential of metformin to exert direct plaque stabilization effects in type 2 diabetic subjects.

Keywords: Diabetes mellitus; plaque; optical coherence tomography (OCT); coronary artery disease (CAD)

Submitted May 30, 2021. Accepted for publication Sep 29, 2021.

doi: $10.21037 /$ cdt-21-346

View this article at: https://dx.doi.org/10.21037/cdt-21-346 


\section{Introduction}

The current therapeutic guidelines from the American Diabetes Association (ADA) and the European Association for the Study of Diabetics (EASD) recommend metformin as the first step for pharmacological treatment of type 2 diabetes $(1,2)$. This is based, in part, on the findings from the UK Prospective Diabetes Study (UKPDS) which demonstrated that metformin reduced diabetic macrovascular complications compared to insulin and sulphonylureas (3). The mechanism underlying any potential benefit of metformin on cardiovascular outcomes in UKPDS trial are unlikely to be due to a glucose lowering effect, given that comparable glucose lowering was observed with other therapies. This observation suggests that metformin may possess additional properties, including direct effects on atherosclerotic plaque, beyond its impact on glycemic control.

Several putative atheroprotective mechanisms of metformin have been reported. Cell and animal studies have demonstrated that metformin favorably modulates secretion of proinflammatory cytokines and AMP-activated kinase (AMPK) implicated in atherogenesis (4-6). Metformin has also been shown to enhance the functional activities of high-density lipoprotein (HDL) $(7,8)$. Given the role that inflammatory cytokines and HDL play in atherogenesis, these findings suggest that metformin may favourably modulate atherosclerotic plaques in patients with type 2 diabetes. Frequency-domain optical coherence tomography (FD-OCT) provides high-resolution intravascular images of plaque components and microstructures in vivo $(9,10)$. In order to gain insights to the probable impact of metformin on the atherosclerotic plaques, we employed FD-OCT imaging in patients with type 2 diabetes and established coronary artery disease (CAD).

We present the following article in accordance with the STROBE reporting checklist (available at https://cdt. amegroups.com/article/view/10.21037/cdt-21-346/rc).

\section{Methods}

\section{Study subjects}

The current study retrospectively analyzed FD-OCT imaging data in type 2 diabetic patients with $\mathrm{CAD}$ requiring percutaneous coronary intervention (PCI) at Cleveland Clinic from May 2011 to April 2014. FD-OCT imaging was used according to operator's discretion. In detail, OCT imaging was not conducted in subjects with the following reasons: angiography or intravascular ultrasound (IVUS)-guided PCI, anatomically unsuitable lesions for FD-OCT imaging (left main disease, chronic total occlusion within target vessel, vessel size $\geq 4 \mathrm{~mm}$, severe tortuosity, severe calcification), chronic kidney disease or worse clinical conditions (congestive heart failure and cardiogenic shock). As a consequence, there were 330 type 2 diabetic subjects with FD-OCT imaging data. Of these, 17 patients were excluded due to poor image quality $(n=14)$ or imaging within a bypass graft $(n=3)$. The remaining 313 patients with CAD were included into the current analysis. The study was conducted in accordance with the Declaration of Helsinki (as revised in 2013). The institutional review board of the Cleveland Clinic approved this retrospective audit of FD-OCT images and clinical data from patients who had undergone FD-OCT imaging during clinically indicated PCI procedures. Because of the retrospective nature of the study, the requirement for informed consent was waived.

\section{FD-OCT imaging procedure}

FD-OCT catheter $\left(\mathrm{C} 7-\mathrm{XR}^{\mathrm{TM}}\right.$ OCT Intravascular Imaging System; St. Jude Medical, St. Paul, Minnesota, USA) was used to visualize the entire target vessel requiring PCI as reported previously $(11,12)$. FD-OCT catheter was automatically withdrawn from the distal to the proximal of the target artery under continuous infusion of contras medium (10-15 mL per vessel). When necessary, multiple FD-OCT pullbacks were conducted to evaluate entire vessel. Side branch was used to analyze overlapped segments of FD-OCT images.

\section{Analysis of FD-OCT images}

The raw FD-OCT imaging data was anonymized and analyzed by using open-source software, ImageJ (National Institutes of Health, Bethesda, Maryland, USA). The current analysis included non-culprit plaques within the target vessel where PCI was conducted. Non-culprit plaque was defined as a coronary artery stenosis in which PCI had not been performed. We analyzed those with diameter stenosis $20-70 \%$ on quantitative coronary angiography. At least 5-mm interval existed between each analyzed plaque. Plaque components and microstructures were analyzed as follows.

\section{Plaque composition}

Lipid component was defined as a low signal region with diffuse border (13). Every frame through the entire length 
of analyzed plaques was analyzed to measure lipid arc. The longitudinal view was used to evaluate lipid length. Lipid index was defined as the mean lipid arc multiplied by lipid length $(14,15)$. Fibrous plaque was defined as the presence of high backscattering and a relatively homogeneous FD-OCT signals (13). Calcification was defined as a signal-poor or heterogeneous region with a sharply delineated border (13).

\section{Plaque microstructures}

The fibrous cap thickness was defined as the minimum distance from the coronary artery lumen to inner border of lipid (13). The averaged value of three measurements at its thinnest part was used for the analysis. Thin-cap fibroatheroma was a plaque containing lipid arc in $\geq 2$ quadrants and the thinnest part of fibrous cap $\leq 65 \mu \mathrm{m}$ (13). A microchannel was defined as a signal-poor void which did not connect to the lumen, recognized on more than 3 consecutive cross-sectional images (13). Cholesterol crystal was defined as a thin, linear region of high signal intensity at a lipidic plaque, accompanied by the absence of signal attenuation $(11,13)$. Intracoronary thrombus was defined as a mass protruding into the vessel lumen from the surface of the vessel wall (13). Plaque rupture was defined as the presence of fibrous cap discontinuity and cavity formation in the plaque (13). Spotty calcification was defined as the presence of lesions $<4 \mathrm{~mm}$ in length and containing an arc of calcification $<90^{\circ}$ according to the definition of previous studies using grayscale IVUS (16).

All FD-OCT images were evaluated at Atherosclerosis Imaging Core Laboratory of Cleveland Clinic by two experienced investigators who were blinded to the clinical presentation and treatment status. When there was discordance between investigators, third independent investigator analyzed the image and then a consensus reading was obtained. Intraclass correlations were 0.92 and 0.98 , 0.98 and 0.99 , and 0.97 and 0.98 for lipid, calcification and fibrous plaques, respectively. Intraobserver and interobserver differences in measurements of fibrous cap thickness and lipid arc were $18 \pm 16$ and $13 \pm 11 \mu \mathrm{m}$, and $23^{\circ} \pm 14^{\circ}$ and $12^{\circ} \pm 10^{\circ}$, respectively. The inter- and intra-observer coefficients and differences for fibrous cap thickness measurements were 0.92 and 0.96 , and $17 \pm 15$ and $12 \pm 10 \mu \mathrm{m}$, respectively. Inter- and intra-observer variabilities yielded acceptable concordance for cholesterol crystal ( $\kappa=0.87$ and 0.89 , respectively) and lipid plaque ( $\kappa=0.89$ and 0.93 , respectively).
Quantitative coronary angiography analysis of non-culprit plaques

Quantitative coronary angiography (CMS-QCA ver 7.0, Medis, Leiden, The Netherlands) analysis was conducted to evaluate reference diameter, minimum lumen diameter and diameter stenosis at non-culprit plaques.

\section{Statistical analysis}

Continuous variables are expressed as mean \pm standard deviation (SD) or median, and categorical variables as percentage. For analysis of patient characteristics, the Chisquare test was used to test for differences in categorical variables between groups and continuous data were compared using unpaired $t$-test, or Mann-Whitney test when the variable was not normally distributed. For the comparison of plaque characteristics between the groups, due to the multiple plaques analyzed within a single patient's data, generalized estimating equations approach was used to take into account its clustering. Averaged lipid arc, and the frequency of cholesterol crystal and spotty calcification were examined using analysis of covariance (ANOCVA), with age, female, metabolic syndrome, chronic kidney disease, low-density lipoprotein cholesterol (LDL-C) and glycated hemoglobin levels as covariates. Subgroup analyses were performed to analyze effects of various clinical characteristics subgroups as well as their interaction effects with metformin use on averaged lipid arc. All statistical analyses were performed using SPSS (version 17.0, SPSS Inc., Chicago, Illinois, USA). $\mathrm{P}<0.05$ was considered significant.

\section{Results}

\section{Clinical demographics}

Of the 313 study subjects, $38.6 \%$ were being treated with metformin at the time of FD-OCT imaging. Clinical characteristics of the patients are summarized in Table 1. Patients treated with metformin more likely had a history of hypertension (79.3\% vs. $67.1 \%, \mathrm{P}=0.03)$, metabolic syndrome $(52.8 \%$ vs. $36.4 \%, \mathrm{P}=0.01)$ and chronic kidney disease $(2.4 \%$ vs. $17.7 \%, \mathrm{P}=0.01)$ compared with patients who were not treated with metformin. There were no significant differences in the frequency of previous myocardial infarction $(\mathrm{P}=0.84)$, stroke $(\mathrm{P}=0.90)$, peripheral 
Table 1 Clinical characteristics

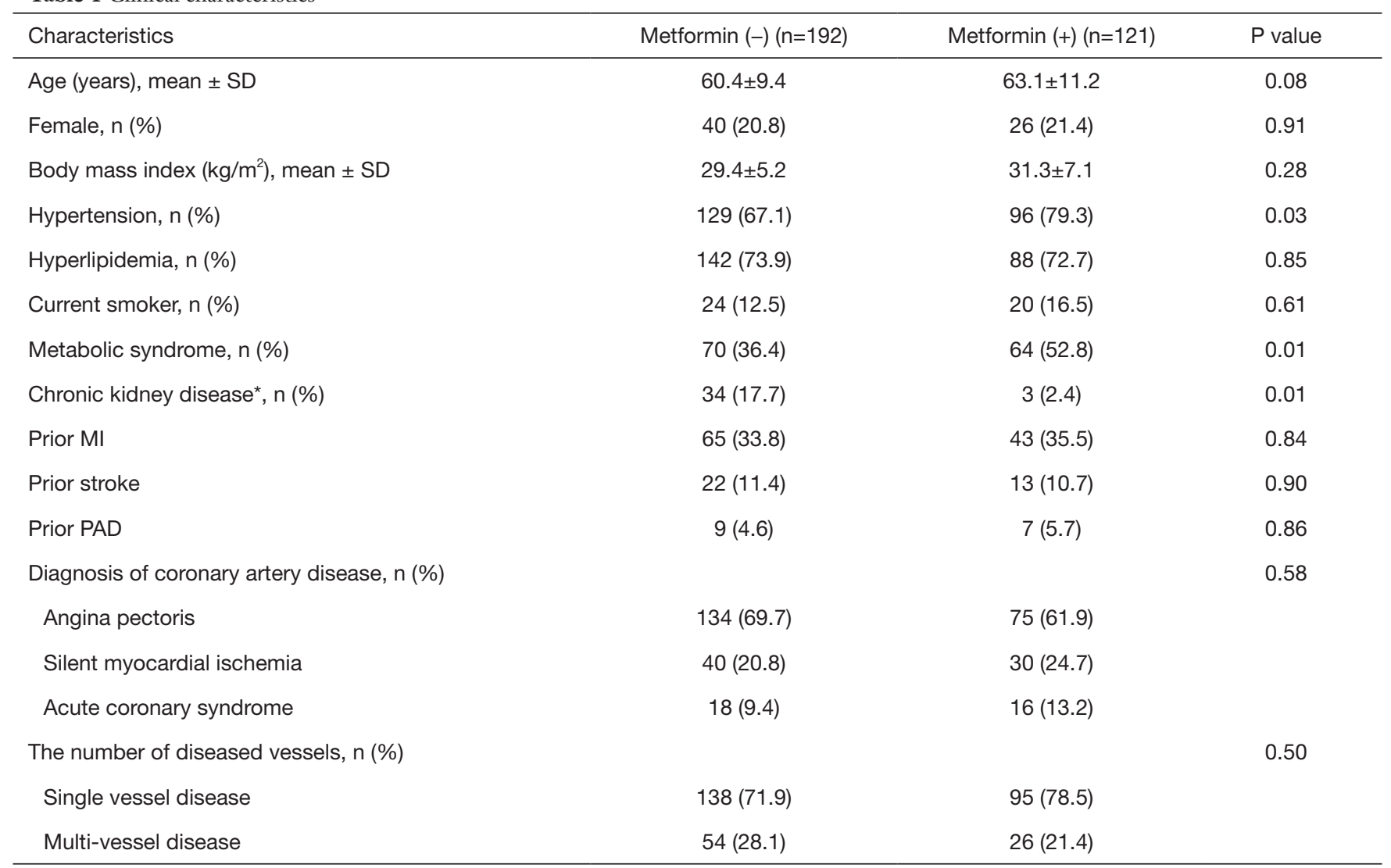

*, chronic kidney disease = estimated glomerular filtration rate $<60 \mathrm{~mL} / \mathrm{min} / 1.73 \mathrm{~m}^{2}$. SD, standard deviation; MI, myocardial infarction; $\mathrm{PAD}$, peripheral artery disease.

artery disease $(\mathrm{P}=0.86)$ and multi-vessel disease $(\mathrm{P}=0.50)$ between the two groups (Table 1).

Table 2 shows medication use and risk factor control. Established medical therapies were used in the two groups with similar frequencies (aspirin: $98.3 \%$ vs. $99.4 \%, \mathrm{P}=0.86$; $\beta$-blocker: $78.5 \%$ vs. $72.9 \%, \mathrm{P}=0.53$; angiotensin converting enzyme inhibitor: $58.6 \%$ vs. $54.1 \%, \mathrm{P}=0.59$; statin: $95.9 \%$ vs. $94.3 \%, \mathrm{P}=0.83$ ) in patients treated with and without metformin, respectively. A low background of use of pioglitazone (17\%) and insulin (15\%) was observed in both groups. Patients treated with metformin demonstrated a lower level of glycated hemoglobin $(7.4 \% \pm 0.5 \% v s$. $8.0 \% \pm 0.6 \%, \mathrm{P}=0.03)$, whereas lipid and blood pressure control were comparable between the two groups (Table 2).

\section{FD-OCT imaging procedure and imaged non-culprit plaques}

In the current study, 216, 79 and 28 cases received single, double and triple vessel FD-OCT imaging, respectively. Throughout these imaging procedures, 559 non-culprit plaques within 458 imaged vessels were identified (Table 3). There was no significant difference in the distribution of single, double and triple vessel imaging $(\mathrm{P}=0.95)$, the number of pullbacks $(2.2 \pm 0.6$ vs. $2.1 \pm 0.5, \mathrm{P}=0.91)$ and the imaged length of coronary artery $(67 \pm 15 \mathrm{vs}$. $64 \pm 14 \mathrm{~mm}, \mathrm{P}=0.85$ ) between patients treated with and without metformin. Plaque composition and anatomical features, in addition to corresponding angiographic measures are also shown in Table 3. A lower prevalence of lipid plaque was observed in patients treated with metformin $(66.2 \%$ vs. $77.9 \%, \mathrm{P}=0.03)$.

\section{Plaque features during metformin use}

Tables 4,5 summarizes measures of lipidic components and microstructures at the non-culprit plaques in the two groups. Non-culprit plaques in patients treated with 
Table 2 Medication use and risk factor control

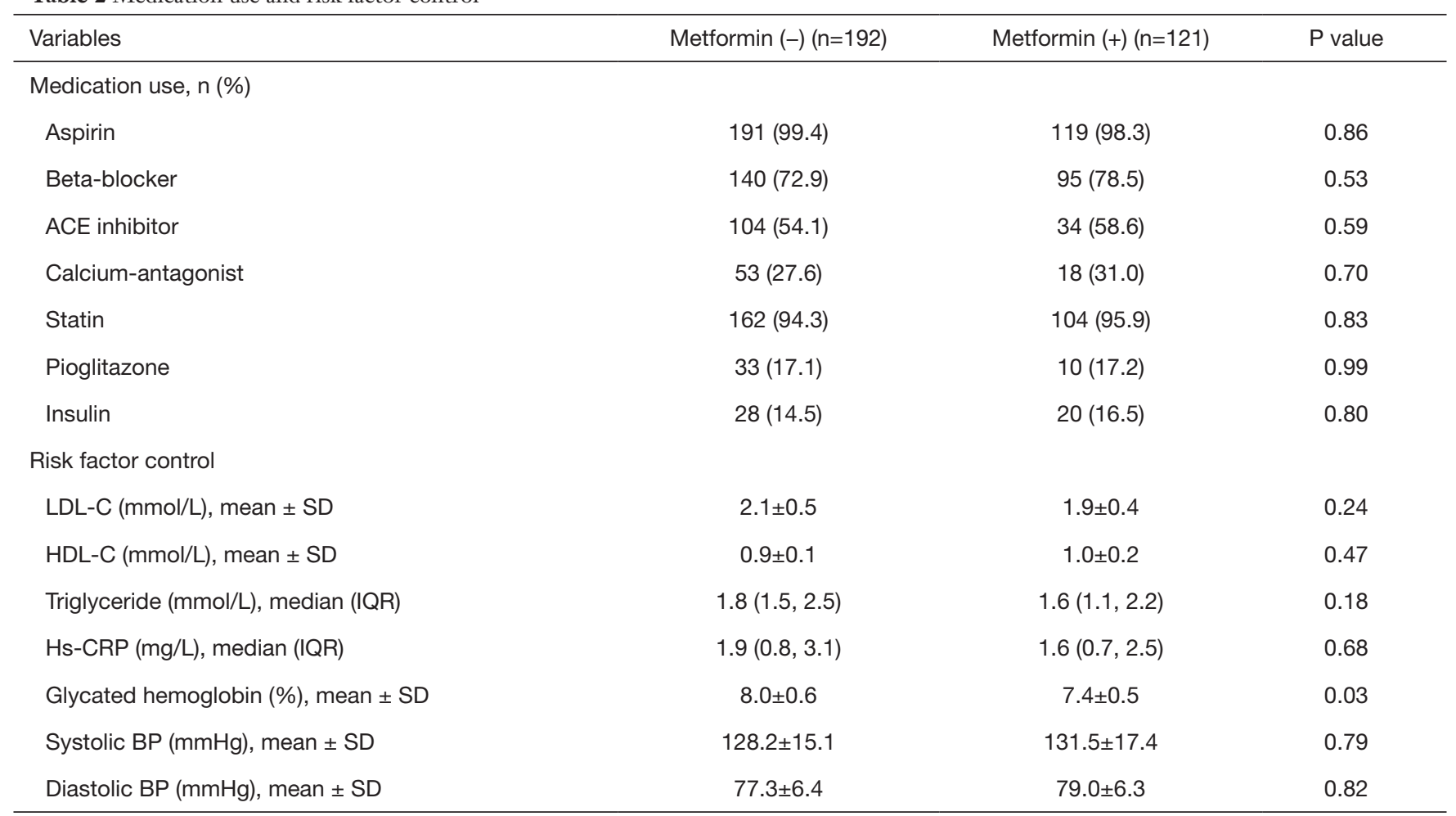

$\mathrm{ACE}$, angiotensin converting enzyme; BP, blood pressure; hs-CRP, c-reactive protein; HDL-C, high-density lipoprotein cholesterol; LDL-C, low-density lipoprotein cholesterol; SD, standard deviation; IQR, interquartile range.

metformin demonstrated a smaller lipid arc (median: $168.7^{\circ}$ vs. $208.5^{\circ}, \mathrm{P}=0.008$ ) and shorter longitudinal length (median: 5.1 vs. $9.1 \mathrm{~mm}, \mathrm{P}=0.04$ ) compared with the group treated without metformin. With regard to plaque microstructures, there were no significant differences between the groups in fibrous cap thickness (median: 114.8 vs. $109.1 \mu \mathrm{m}, \mathrm{P}=0.52$ ), the prevalence of thin-cap fibroatheroma $(8.6 \%$ vs. $10.1 \%, \mathrm{P}=0.85)$, microchannel (19.2\% vs. $20.1 \%, \mathrm{P}=0.95)$, plaque rupture (2.6\% vs. $2.7 \%$, $\mathrm{P}=0.98)$ and thrombus $(2.6 \%$ vs. $3.1 \%, \mathrm{P}=0.98)$. In contrast, metformin treated patients demonstrated a lower frequency of cholesterol crystal $(3.9 \%$ vs. $18.2 \%, \mathrm{P}=0.01)$ and spotty calcification $(3.9 \%$ vs. $34.8 \%, \mathrm{P}=0.008)$ compared with patients not treated with metformin. Following adjustment for clinical characteristics (age, gender, metabolic syndrome and chronic kidney disease), statin use and the degree of risk factor control (LDL-C and glycated hemoglobin levels), non-culprit plaques in patients treated with metformin still continued to exhibit smaller lipid arc (median: $163.4^{\circ} \mathrm{vs}$. $193.5^{\circ}, \mathrm{P}=0.02$ ), and lower frequency of cholesterol crystal (2.4\% vs. $14.5 \%, \mathrm{P}=0.03)$ and spotty calcification $(6.5 \%$ vs. $23.2 \%, \mathrm{P}=0.01)$ compared with patients who were not treated with metformin (Tables 4,5). Two representative cases were shown by Figure 1 .

The relationship between metformin use and averaged lipid arc in sub-groups is shown in Table 6. In most subgroups, a smaller lipid arc was observed in patients who were treated with metformin compared with those who were not (Table 6). However, in patients who received insulin, the use of metformin was not associated with a smaller lipid arc [insulin use (+): $194.6^{\circ}$ vs. $207.4^{\circ}, \mathrm{P}=0.87$; insulin use (-): $156.7^{\circ}$ vs. $204.3^{\circ}, \mathrm{P}=0.009, \mathrm{P}$ value for interaction between two groups, $\mathrm{P}=0.02$; Table 6).

\section{Discussion}

High resolution FD-OCT imaging has provided mechanistic insights into the potential efficacy of antiatherosclerotic medical therapies in vivo. In the current study, non-culprit coronary lesions in patients with type 2 diabetes receiving metformin demonstrated evidence of greater plaque stabilization, reflected by the presence of a smaller lipid arc and a lower frequency of both cholesterol crystals and spotty calcification. These differences 
Table 3 Comparisons of imaged vessels and plaques

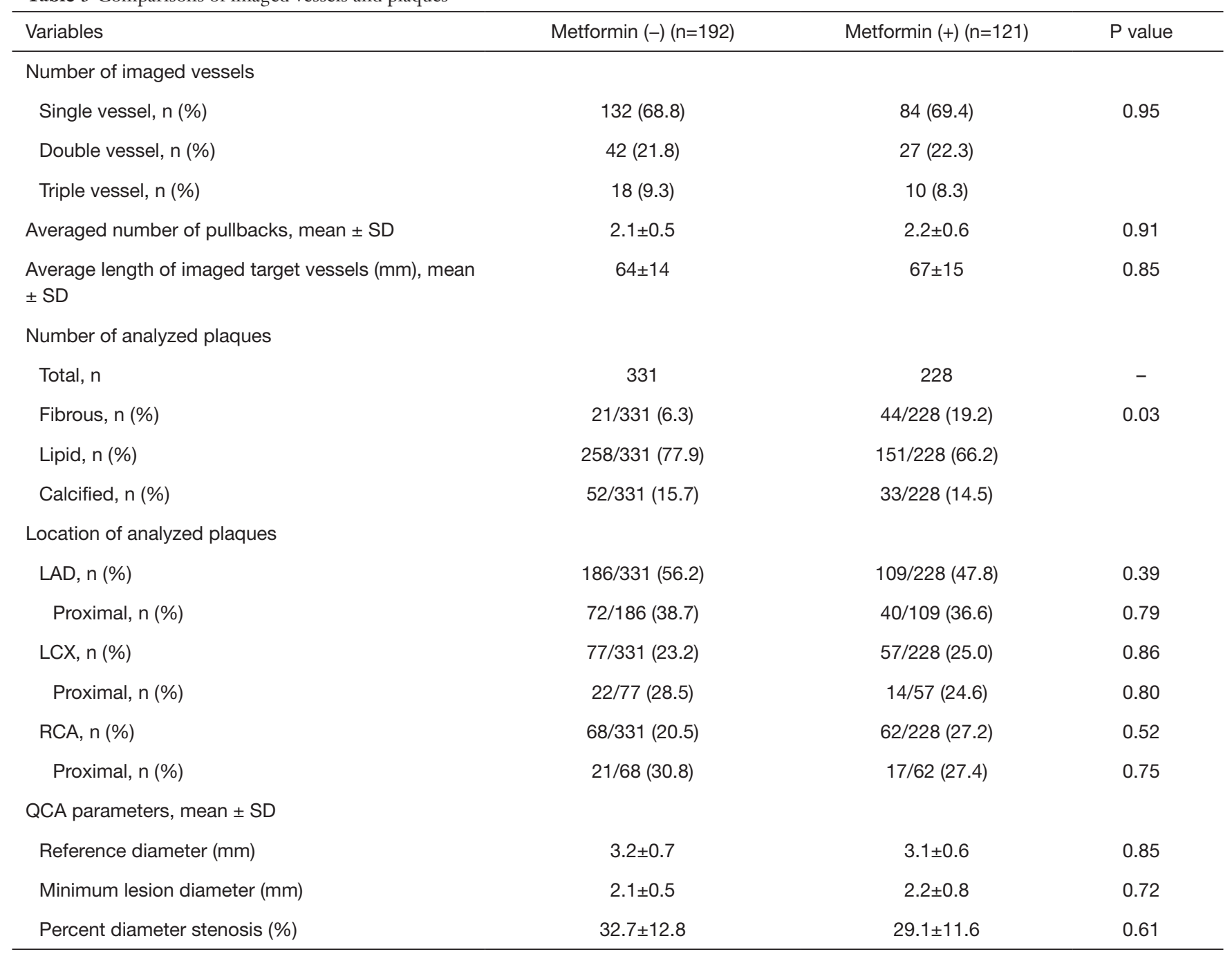

FD-OCT, frequency-domain optical coherence tomography; LAD, left anterior descending artery; LCX, left circumflex artery; RCA, right coronary artery; QCA, quantitative coronary angiography; SD, standard deviation.

between the groups persisted after adjusting for clinical characteristics including glycemic control and LDL-C levels. These findings suggest that metformin may have the potential to favourably modulate atherosclerotic plaques, in a manner independent from glucose lowering.

To date, there is no clinical study which evaluated characteristics of coronary atherosclerosis in type 2 diabetic patients treated with metformin. In our analysis, FDOCT measures of lipid components at non-culprit plaques were less prevalent in patients treated with metformin. Mechanistic studies have suggested that the extent of lipidic material could be modified by metformin, with evidence of reduced macrophage content within the atherosclerotic plaques of $\mathrm{ApoE}^{-/-}$mice in association with AMPK activation (17). Metformin has also been demonstrated to inhibit NF- $\kappa \mathrm{B}$ signaling, an important factor in the stimulation of inflammatory events and foam cell formation within the artery wall (4). Additional ex vivo studies have demonstrated that enhanced HDL-mediated cholesterol efflux capacity from macrophages in the setting of metformin treatment $(7,8)$. Given the importance of these factors in regulating the development of lipidic lesions within the artery wall, the findings suggest the potential for metformin to exert anti-atherosclerotic effects beyond its role in glucose lowering.

A number of additional observations were made 
Table 4 Lesion-based comparison of FD-OCT measures at lipid plaques

\begin{tabular}{|c|c|c|c|}
\hline Variables & Metformin $(-)(n=258)$ & Metformin $(+)(n=151)$ & $P$ value \\
\hline Averaged lipid $\operatorname{arc}\left({ }^{\circ}\right)$ & $208.5(136.4,279.6)$ & $168.7(112.5,213.1)$ & 0.008 \\
\hline Longitudinal lipid length (mm) & $9.1(3.4,15.1)$ & $5.1(1.5,8.4)$ & 0.04 \\
\hline \multicolumn{4}{|l|}{ Plaque microstructures } \\
\hline TCFA, n (\%) & $26(10.1)$ & $13(8.6)$ & 0.85 \\
\hline Microchannel, n (\%) & $52(20.1)$ & $29(19.2)$ & 0.95 \\
\hline Cholesterol crystal, n (\%) & $47(18.2)$ & $6(3.9)$ & 0.01 \\
\hline Spotty calcification, n (\%) & $90(34.8)$ & $6(3.9)$ & 0.008 \\
\hline
\end{tabular}

FD-OCT, frequency-domain optical coherence tomography; TCFA, thin-cap fibroatheroma; IQR, interquartile range.

Table 5 Comparison of FD-OCT measures adjusting for clinical characteristics

\begin{tabular}{|c|c|c|c|}
\hline Variables & Metformin (-) & Metformin (+) & $P$ value \\
\hline Cholesterol crystal $(\%)^{*}$ & 14.5 & 2.4 & 0.03 \\
\hline Spotty calcification $(\%)^{\star}$ & 23.2 & 6.5 & 0.01 \\
\hline
\end{tabular}

*, the presented values are adjusted for age, gender, metabolic syndrome, chronic kidney disease, statin use, LDL-C and glycated hemoglobin levels. FD-OCT, frequency-domain optical coherence tomography; LDL-C, low-density lipoprotein cholesterol; IQR, interquartile range.

favoring a potential anti-atherosclerotic effect of metformin. The lower prevalence of cholesterol crystals in the metformin group is important, given their ability of physically penetrating fibrous caps and stimulating a local inflammatory response via NLRP3 inflammasome activation, both of which are known mediators of plaque instability (18-20). The presence of relatively lipid poor plaques in coupled with metformin's favourable impact on cholesterol efflux capacity via HDL and macrophage activation, may be one of the mechanisms by which metformin reduce cholesterol crystallization and the consequent inflammatory activity. The potential of metformin to stabilize coronary plaques via a reduction in cholesterol crystal formation requires further investigation in future studies.

Spotty calcification is an important driver to induce accelerated progression and instability of coronary atherosclerotic plaques $(16,21,22)$. In this study, we observed that non-culprit lesions were less likely to contain spotty calcification in the setting of metformin use. This finding further supports plaque stabilizing effect of metformin possibly via reductions in plaque inflammation and resultant spotty calcification. A recent study has demonstrated that AMPK $\alpha 1$ activation is negatively associated with osteoblastic differentiation of VSMC in vitro (23). In addition, metformin markedly inhibited calcium formation in atherosclerotic plaque of aortic roots in $\mathrm{ApoE}^{-/-}$mice via its activation of AMPK $\alpha$ (23). Our findings on OCT along with the aforementioned mechanisms may indicate the distinct property of metformin to modulate calcific components within the plaques.

Thin-cap fibroatheroma is an important signature of coronary atheroma causing future coronary events. In the current study, the frequency of this plaque phenotype did not differ between subjects with and without metformin. Another OCT imaging study reported that lowering 

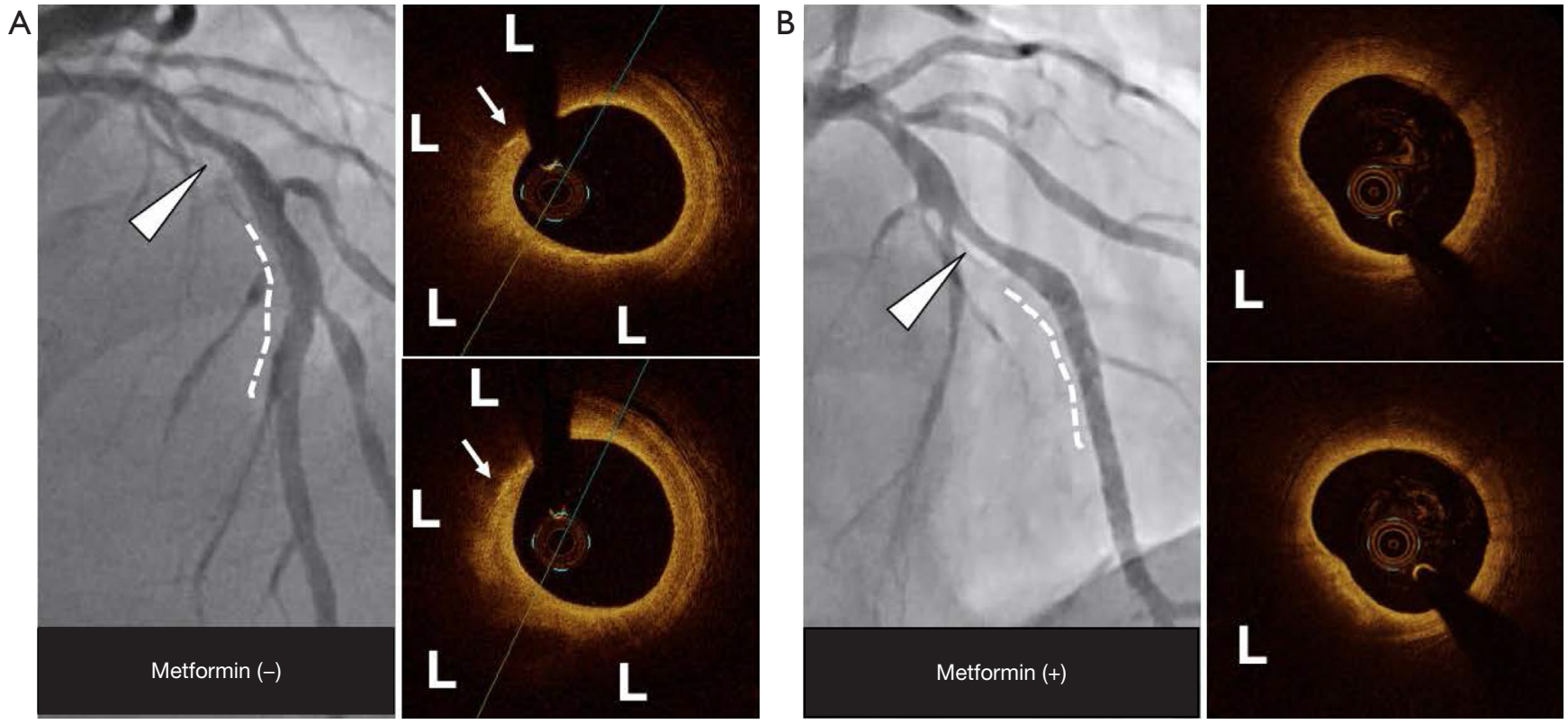

Figure 1 Representative cases. (A) A 65-year-old gentleman received percutaneous coronary intervention (PCI) at the middle segment of hid left anterior descending artery (white dotted line). He has not taken metformin prior to PCI. optical coherence tomography (OCT) imaging at non-culprit segment (white arrow head) demonstrated a large lipid-rich plaque (L) containing cholesterol crystal (white arrow). (B) A 50-year gentleman was hospitalized due to anginal chest pain. Stent implantation was conducted at the middle segment of hid left anterior descending artery (white dotted line). He was treated by metformin. On OCT imaging, non-culprit segment (white arrow head) exhibited a small lipid plaque (L) without any cholesterol crystal.

$\mathrm{LDL}-\mathrm{C}<50 \mathrm{mg} / \mathrm{dL}$ was associated with thicker fibrous cap in non-diabetic but not diabetic subjects (24), suggesting that thin fibrous cap may not necessarily respond well to risk factor control. Inflammation is one of drivers to affect fibrous cap thickness. Recent studies showed antiinflammatory action of metformin $(25,26)$. The potency of metformin-mediated anti-inflammatory effect may not be enough to modulate thin-cap fibroatheroma. Since the current study did not measure inflammatory markers, it remains unknown the association of inflammatory activity with the frequency of thin-cap fibroatheroma under metformin use.

While smaller lipid arc under metformin therapy was consistently observed in a variety of subgroups, this feature was not identified in type 2 diabetic subjects taking insulin. Recent randomized clinical trial also demonstrated that metformin use did not necessarily reduce coronary events in diabetic subjects receiving insulin (27). The exact mechanism of these findings remains to be determined. However, since patients requiring insulin therapy typically have a longer duration of type 2 diabetes mellitus and more severe hyperglycemia, the longer exposure to hyperglycemia may promote the deposition of fibrotic and calcific materials (28-30), which may diminish the response to metformin therapy. Diabetic patients receiving insulin are more likely to exhibit negative remodeling (31). Given that greater atheroma burden with positive remodeling is associated with favourable response to anti-atherosclerotic medical therapy (32-34), this morphological feature may suggest that its modifiability would be attenuated under the therapy.

A number of caveats of the present analysis should be considered. The current study did not collect data about duration and dose of metformin use. We cannot analyze how much treatment duration and dose of metformin affects OCT features. The current analyses included clinically indicated FD-OCT imaging. In particular, the current study mainly included stable CAD receiving OCT imaging. Due to achieving prompt reperfusion, operators may more likely perform primary PCI without the use of OCT imaging. These may be a cause for selection bias. Due to the shallow penetration depth of the infra-red light beam, FD-OCT is not fully capable of evaluating the full burden of plaque within vessel wall. Furthermore, the study was performed only in patients with an established clinical diagnosis of 
Table 6 Sub-group analysis of averaged lipid arc under metformin use

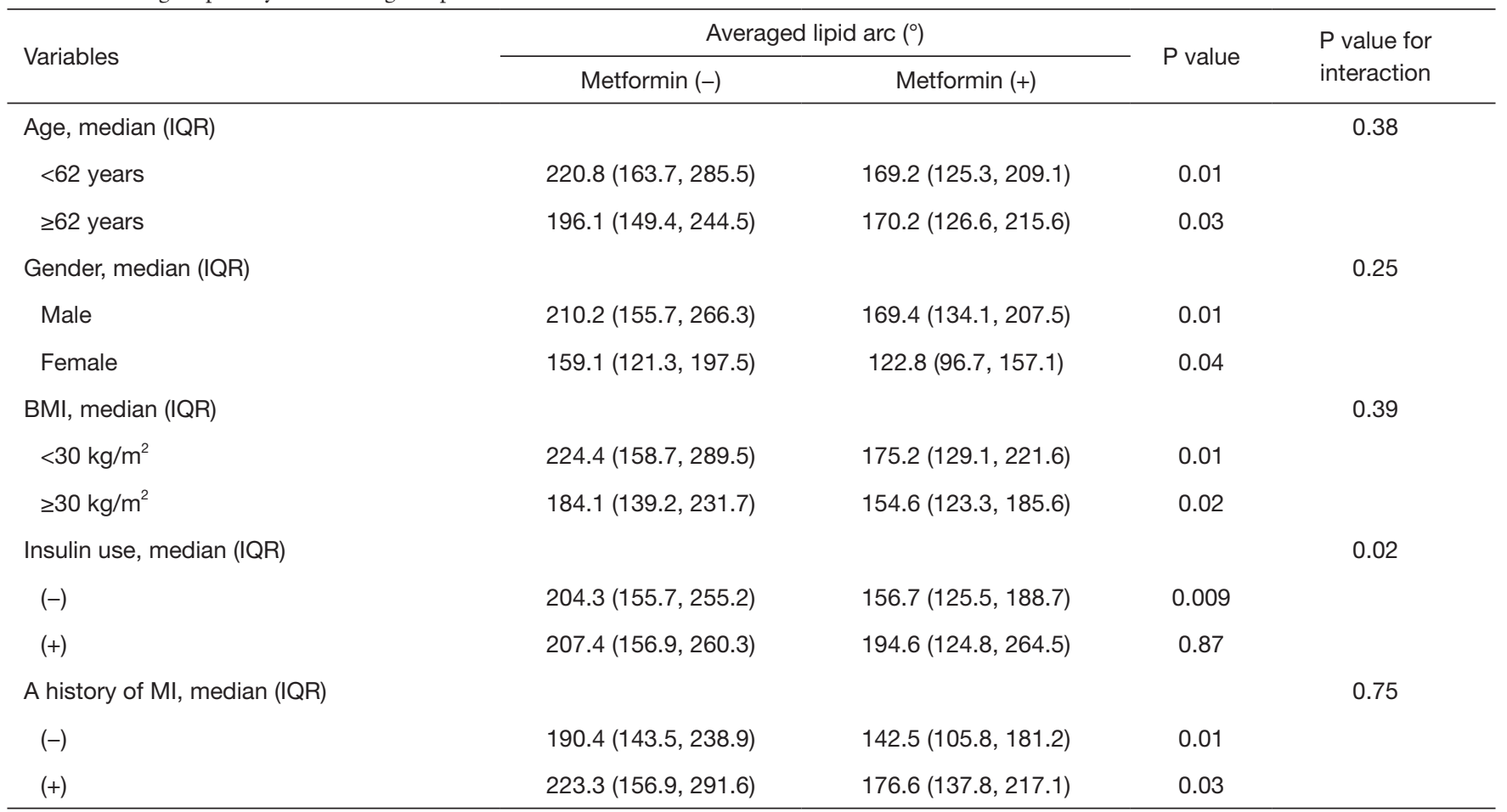

BMI, body mass index; HbA1c, glycated hemoglobin; LDL-C, low-density lipoprotein cholesterol; MI, myocardial infarction; IQR, interquartile range.

type 2 diabetes. With increasing use of metformin in patients with prediabetes, it is unknown whether similar effects would be observed. This study is a hypothesis generating one, which warrants future investigation.

In summary, the use of metformin was associated with smaller lipid arc, and a lower frequency of cholesterol crystals and spotty calcification at non-culprit plaques in type 2 diabetic patients with CAD. Despite adjusting clinical, lipoprotein and glycemic parameters, favorable features of plaque microstructures under metformin use on FD-OCT imaging suggest a distinct property of metformin to stabilize atherosclerotic plaques in type 2 diabetic patients, which may account for its reduction of diabetic macrovascular disease including CAD.

\section{Acknowledgments}

The authors are grateful for the technical expertise of the Atherosclerosis Imaging Core Laboratory of the Cleveland Clinic.

Funding: None.

\section{Footnote}

Reporting Checklist: The authors have completed the STROBE reporting checklist. Available at https://cdt. amegroups.com/article/view/10.21037/cdt-21-346/rc

Data Sharing Statement: Available at https://cdt.amegroups. com/article/view/10.21037/cdt-21-346/dss

Conflicts of Interest: All authors have completed the ICMJE uniform disclosure form (available at https://cdt.amegroups. com/article/view/10.21037/cdt-21-346/coif). YK has received research support from Nipro and Abbott, and honoraria from Nipro, Abbott, Kowa, Amgen, Sanofi, Astellas, Takeda and Daiichi-Sankyo. SJN is a consultant for AstraZeneca, Amgen, Boehringer Ingelheim, CSL Behring, Merck, Takeda, Novartis, Pfizer, Sanofi-Regeneron, Eli Lilly and Roche and has received research support from AstraZeneca, Amgen, Cerenis, Novartis, Resverlogix, Eli Lilly, InfraReDx, Anthera, Roche, Sanofi-Regeneron and Lipid Sciences. KU was an employee of Sanofi before joining 
this study. SEN has received research support to perform clinical trials through the Cleveland Clinic Coordinating Center for Clinical Research from Pfizer, AstraZeneca, Novartis, Roche, Daiichi-Sankyo, Takeda, Sanofi-Aventis, Resverlogix, and Eli Lilly; and is a consultant/advisor for many pharmaceutical companies but requires them to donate all honoraria or consulting fees directly to charity so that he receives neither income nor a tax deduction. YK and SJN serve as unpaid editorial board members of Cardiovascular Diagnosis and Therapy from Jul 2019 to Jun 2021. The other authors have no conflicts of interest to declare.

Ethical Statement: The authors are accountable for all aspects of the work in ensuring that questions related to the accuracy or integrity of any part of the work are appropriately investigated and resolved. The study was conducted in accordance with the Declaration of Helsinki (as revised in 2013). The institutional review board of the Cleveland Clinic approved this retrospective audit of FD-OCT images and clinical data from patients who had undergone FD-OCT imaging during clinically indicated PCI procedures. Because of the retrospective nature of the study, the requirement for informed consent was waived.

Open Access Statement: This is an Open Access article distributed in accordance with the Creative Commons Attribution-NonCommercial-NoDerivs 4.0 International License (CC BY-NC-ND 4.0), which permits the noncommercial replication and distribution of the article with the strict proviso that no changes or edits are made and the original work is properly cited (including links to both the formal publication through the relevant DOI and the license). See: https://creativecommons.org/licenses/by-nc-nd/4.0/.

\section{References}

1. American Diabetes Association. 8. Pharmacologic Approaches to Glycemic Treatment: Standards of Medical Care in Diabetes-2018. Diabetes Care 2018;41:S73-85.

2. Authors/Task Force Members; Rydén L, Grant PJ, et al. ESC Guidelines on diabetes, pre-diabetes, and cardiovascular diseases developed in collaboration with the EASD: the Task Force on diabetes, pre-diabetes, and cardiovascular diseases of the European Society of Cardiology (ESC) and developed in collaboration with the European Association for the Study of Diabetes (EASD). Eur Heart J 2013;34:3035-87.

3. Effect of intensive blood-glucose control with metformin on complications in overweight patients with type 2 diabetes (UKPDS 34). UK Prospective Diabetes Study (UKPDS) Group. Lancet 1998;352:854-65.

4. Cameron AR, Morrison VL, Levin D, et al. AntiInflammatory Effects of Metformin Irrespective of Diabetes Status. Circ Res 2016;119:652-65.

5. Ramachandran S, Anandan V, Kutty VR, et al. Metformin attenuates effects of cyclophilin A on macrophages, reduces lipid uptake and secretion of cytokines by repressing decreased AMPK activity. Clin Sci (Lond) 2018;132:719-38.

6. Vasamsetti SB, Karnewar S, Kanugula AK, et al. Metformin inhibits monocyte-to-macrophage differentiation via AMPK-mediated inhibition of STAT3 activation: potential role in atherosclerosis. Diabetes 2015;64:2028-41.

7. Ma A, Wang J, Yang L, et al. AMPK activation enhances the anti-atherogenic effects of high density lipoproteins in apoE-/- mice. J Lipid Res 2017;58:1536-47.

8. Matsuki K, Tamasawa N, Yamashita M, et al. Metformin restores impaired HDL-mediated cholesterol efflux due to glycation. Atherosclerosis 2009;206:434-8.

9. Huang D, Swanson EA, Lin CP, et al. Optical coherence tomography. Science 1991;254:1178-81.

10. Jang IK, Bouma BE, Kang DH, et al. Visualization of coronary atherosclerotic plaques in patients using optical coherence tomography: comparison with intravascular ultrasound. J Am Coll Cardiol 2002;39:604-9.

11. Kataoka Y, Puri R, Hammadah M, et al. Cholesterol crystals associate with coronary plaque vulnerability in vivo. J Am Coll Cardiol 2015;65:630-2.

12. Kataoka Y, Puri R, Hammadah M, et al. Sex Differences in Nonculprit Coronary Plaque Microstructures on Frequency-Domain Optical Coherence Tomography in Acute Coronary Syndromes and Stable Coronary Artery Disease. Circ Cardiovasc Imaging 2016;9:e004506.

13. Tearney GJ, Regar E, Akasaka T, et al. Consensus standards for acquisition, measurement, and reporting of intravascular optical coherence tomography studies: a report from the International Working Group for Intravascular Optical Coherence Tomography Standardization and Validation. J Am Coll Cardiol 2012;59:1058-72.

14. Kato K, Yonetsu T, Kim SJ, et al. Comparison of nonculprit coronary plaque characteristics between patients with and without diabetes: a 3-vessel optical coherence tomography study. JACC Cardiovasc Interv 2012;5:1150-8.

15. Kato K, Yonetsu T, Jia H, et al. Nonculprit coronary 
plaque characteristics of chronic kidney disease. Circ

Cardiovasc Imaging 2013;6:448-56.

16. Kataoka Y, Puri R, Hammadah M, et al. Spotty calcification and plaque vulnerability in vivo: frequencydomain optical coherence tomography analysis. Cardiovasc Diagn Ther 2014;4:460-9.

17. Wang J, Ma A, Zhao M, et al. AMPK activation reduces the number of atheromata macrophages in ApoE deficient mice. Atherosclerosis 2017;258:97-107.

18. Janoudi A, Shamoun FE, Kalavakunta JK, et al. Cholesterol crystal induced arterial inflammation and destabilization of atherosclerotic plaque. Eur Heart J 2016;37:1959-67.

19. Abela GS, Aziz K, Vedre A, et al. Effect of cholesterol crystals on plaques and intima in arteries of patients with acute coronary and cerebrovascular syndromes. Am J Cardiol 2009;103:959-68.

20. Duewell P, Kono H, Rayner KJ, et al. NLRP3 inflammasomes are required for atherogenesis and activated by cholesterol crystals. Nature 2010;464:1357-61.

21. Kataoka Y, Wolski K, Uno K, et al. Spotty calcification as a marker of accelerated progression of coronary atherosclerosis: insights from serial intravascular ultrasound. J Am Coll Cardiol 2012;59:1592-7.

22. Nakahara T, Dweck MR, Narula N, et al. Coronary Artery Calcification: From Mechanism to Molecular Imaging. JACC Cardiovasc Imaging 2017;10:582-93.

23. Cai Z, Ding Y, Zhang M, et al. Ablation of Adenosine Monophosphate-Activated Protein Kinase $\alpha 1$ in Vascular Smooth Muscle Cells Promotes DietInduced Atherosclerotic Calcification In Vivo. Circ Res 2016;119:422-33.

24. Kataoka Y, Hammadah M, Puri R, et al. Plaque microstructures in patients with coronary artery disease who achieved very low low-density lipoprotein cholesterol levels. Atherosclerosis 2015;242:490-5.

25. Saisho Y. Metformin and Inflammation: Its Potential Beyond Glucose-lowering Effect. Endocr Metab Immune Disord Drug Targets 2015;15:196-205.

Cite this article as: Kataoka Y, Nicholls SJ, Andrews J, Uno K, Kapadia SR, Tuzcu EM, Nissen SE, Puri R. Plaque microstructures during metformin therapy in type 2 diabetic subjects with coronary artery disease: optical coherence tomography analysis. Cardiovasc Diagn Ther 2022;12(1):77-87. doi: $10.21037 / \mathrm{cdt}-21-346$
26. Bharath LP, Nikolajczyk BS. The intersection of metformin and inflammation. Am J Physiol Cell Physiol 2021;320:C873-9.

27. Kooy A, de Jager J, Lehert P, et al. Long-term effects of metformin on metabolism and microvascular and macrovascular disease in patients with type 2 diabetes mellitus. Arch Intern Med 2009;169:616-25.

28. Portik-Dobos V, Anstadt MP, Hutchinson J, et al. Evidence for a matrix metalloproteinase induction/activation system in arterial vasculature and decreased synthesis and activity in diabetes. Diabetes 2002;51:3063-8.

29. Rumble JR, Cooper ME, Soulis T, et al. Vascular hypertrophy in experimental diabetes. Role of advanced glycation end products. J Clin Invest 1997;99:1016-27.

30. Anand DV, Lim E, Darko D, et al. Determinants of progression of coronary artery calcification in type 2 diabetes role of glycemic control and inflammatory/ vascular calcification markers. J Am Coll Cardiol 2007;50:2218-25.

31. Nicholls SJ, Tuzcu EM, Kalidindi S, et al. Effect of diabetes on progression of coronary atherosclerosis and arterial remodeling: a pooled analysis of 5 intravascular ultrasound trials. J Am Coll Cardiol 2008;52:255-62.

32. Puri R, Nissen SE, Shao M, et al. Antiatherosclerotic effects of long-term maximally intensive statin therapy after acute coronary syndrome: insights from Study of Coronary Atheroma by Intravascular Ultrasound: Effect of Rosuvastatin Versus Atorvastatin. Arterioscler Thromb Vasc Biol 2014;34:2465-72.

33. Kataoka Y, Andrews J, Duong M, et al. Regression of coronary atherosclerosis with infusions of the highdensity lipoprotein mimetic CER-001 in patients with more extensive plaque burden. Cardiovasc Diagn Ther 2017;7:252-63.

34. Kataoka Y, Wolski K, Balog C, et al. Progression of coronary atherosclerosis in stable patients with ultrasonic features of high-risk plaques. Eur Heart J Cardiovasc Imaging 2014;15:1035-41. 